\title{
Fuzzy Implementation of Qubits Operators
}

Alexander Rybalov ${ }^{1}$, Eugene Kagan ${ }^{2}$, Alon Rapoport ${ }^{3}$ and Irad Ben-Gal ${ }^{4 *}$

${ }^{1}$ Jerusalem College of Technology, Jerusalem, 91160, Israel

${ }^{2}$ Department of Industrial Engineering, Ariel University, Ariel, 40700, Israel

${ }^{3}$ Department of Mechanical Engineering, Tel-Aviv University, Ramat-Aviv, 69978, Israel

${ }^{4}$ Department of Industrial Engineering, Tel-Aviv University, Ramat-Aviv, 69978, Israel

\begin{abstract}
In the paper, a complete minimal system of fuzzy logic operators that implements the operations over qubits is suggested. The model is based on a parametric system of the fuzzy not and phase operators and represents operators of quantum information theory acting on real amplitudes. An example of the system application is provided by its implementation to a mobile robot control, and its correspondence with the previously suggested models is illustrated by numerical simulations.
\end{abstract}

Keywords: Quantum information; Fuzzy logic; Mobile robots.

\section{Introduction}

Following the publication of the classical work by Birkhoff and von Neumann [1], the correspondence between the logic of quantum mechanics and non-Boolean logics has attracted significant theoretical and practical interest [2]. During the last decades, such an interest was enhanced with the development of quantum information and computation theory [3] and several approaches for the implementation of quantum logic by the use of fuzzy methods were presented[4-8]. The main goal of these studies is to construct a fuzzy model of quantum mechanical operators such that it can be implemented by fuzzy controllers [9]. In some cases, such models are complementary to the implementation of fuzzy control models by the use of quantum computations [10].

Recently, Hannachi et al. [5,6] suggested a direct fuzzy model of quantum computations over the qubits with real amplitudes. In their model, termed here as the $\mathrm{H}^{3}$-model, qubits are represented by pairs of membership functions, and main quantum gates are implemented by corresponding fuzzy operators on the unit square. Rybalov et al. [11] supplement the $\mathrm{H}^{3}$-model with reverse fuzzy Hadamard operator and implemented the resulting model in a mobile robot control system. The resulting model is indicated henceforth as the extended $\mathrm{H}^{3}$-model.

Although the extended $\mathrm{H}^{3}$-model provides basic representations of quantum information concepts by fuzzy logic methods and, in the completed form, can be used in certain applications, no general and minimal two-dimensional fuzzy model have been reported so far.

The objective of the paper is to present a minimal fuzzy model on a unit square that generalises the extended $\mathrm{H}^{3}$-model and, similar to quantum-mechanical models, preserves smoothness of membership functions and operators. The suggested model applies a parametric system of the fuzzy not and phase operators, such that their compositions provide a complete system of fuzzy operators and implement operators of quantum information theory for real amplitudes. The fuzzy not and phase operators are based on the uninorms [12].

The actions of the suggested minimal fuzzy model are illustrated by the use of a real mobile robot control system. In addition, numerical simulations and comparison with the control method based on the extended $\mathrm{H}^{3}$-model [11] are provided.

The obtained results can support a development of fuzzy logic tools for the models of quantum information theory and their implementation.
The paper is organized as follows. The background includes a brief presentation of the main fuzzy operators, which are included in the $\mathrm{H}^{3}$-model and extended $\mathrm{H}^{3}$-model. In Phase ${ }_{\alpha}$ and not ${ }_{\alpha}$ operators for $\mathrm{H}^{3}$-model, we defined the suggested parametric fuzzy not and phase operators and consider their actions. Uninorm for Phase ${ }_{a}$ and Not Operators and Projector section deals with the uninorm and the metrics for the suggested operators. In implementation of the suggested model and numerical analysis, an example, which illustrates an implementation of the suggested model for the mobile robot's control, is presented. In addition, the section includes results of numerical simulations and statistical comparison of the suggested model with the extended $\mathrm{H}^{3}$-model applied for the mobile robot's control.

\section{Background}

The states $s$ of quantum-mechanical system [3] are defined by the use of qubits that are two elements column-vectors

$$
|s\rangle=(a, b)^{T}=a|0\rangle+b|1\rangle=a(1,0)^{T}+b(0,1)^{T}
$$

where vectors $|0\rangle=(1,0)^{T}$ and $|1\rangle=(0,1)^{T}$ are the basic qubits and $|a|^{2}+|b|^{2}=1$. The basic states $|0\rangle$ and $|1\rangle$ of the system are usually

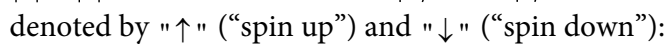

$$
" \uparrow "=\left|s_{1}\right\rangle=(1,0)^{T}, \quad \quad \downarrow "=\left|s_{2}\right\rangle=(0,1)^{T},
$$

while the states " $\leftarrow$ " and $" \rightarrow$ " are defined as follows:

$$
" \rightarrow "=\left|s_{3}\right\rangle=\left(\frac{1}{\sqrt{2}}, \frac{1}{\sqrt{2}}\right)^{T}, " \leftarrow "=\left|s_{4}\right\rangle=\left(\frac{1}{\sqrt{2}},-\frac{1}{\sqrt{2}}\right)^{T} \text {. }
$$

The states $\left|s_{1}\right\rangle,\left|s_{2}\right\rangle,\left|s_{3}\right\rangle$ and $\left|s_{4}\right\rangle$ form a complete system in such a sense that each state can be obtained by the use of the other states [11], i.e.:

$$
\left|s_{1}\right\rangle=\frac{1}{\sqrt{2}}\left|s_{3}\right\rangle+\frac{1}{\sqrt{2}}\left|s_{4}\right\rangle, \quad\left|s_{2}\right\rangle=\frac{1}{\sqrt{2}}\left|s_{3}\right\rangle-\frac{1}{\sqrt{2}}\left|s_{4}\right\rangle,
$$

*Corresponding author: Irad Ben-Gal, Department of Industrial Engineering, TelAviv University, Ramat-Aviv, 69978, Israel, Tel: 13126136968; E-mail: bengal@tau.ac.il

Received June 03, 2014; Accepted June 25, 2014; Published June 27, 2014

Citation: Rybalov A, Kagan E, Rapoport A, Ben-Gal I (2014) Fuzzy Implementation of Qubits Operators. J Comput Sci Syst Biol 7: 163-168. doi:10.4172/jcsb.1000151

Copyright: $\odot 2014$ Rybalov A, et al. This is an open-access article distributed under the terms of the Creative Commons Attribution License, which permits unrestricted use, distribution, and reproduction in any medium, provided the original author and source are credited. 


$$
\left|s_{3}\right\rangle=\frac{1}{\sqrt{2}}\left|s_{1}\right\rangle+\frac{1}{\sqrt{2}}\left|s_{2}\right\rangle, \quad\left|s_{4}\right\rangle=\frac{1}{\sqrt{2}}\left|s_{1}\right\rangle-\frac{1}{\sqrt{2}}\left|s_{2}\right\rangle,
$$

and, as it is usual in quantum mechanics, the states $|s\rangle$ and $(-1) \cdot|s\rangle$ are not distinguished.

According to the Hannachi et al. [5,6], in the direct fuzzy model of quantum computations to each quantum state $|s\rangle=(a, b)^{T}$ with real amplitudes fuzzy state $\tilde{s}=\left(\mu_{a}, \mu_{b}\right)$, in which the membership functions $\mu_{a}:[0,1] \rightarrow[0,1]$ and $\mu_{b}:[0,1] \rightarrow[0,1]$ are defined as follows:

$$
\mu_{a}=\frac{2}{\pi} \arcsin \sqrt{\frac{1}{2}\left(\operatorname{sign}(a) a^{2}-\operatorname{sign}(b) b^{2}+1\right)}, \mu_{b}=\frac{2}{\pi} \arcsin \sqrt{\frac{1}{2}\left(\operatorname{sign}(a) a^{2}+\operatorname{sign}(b) b^{2}+1\right)} .
$$

Inversely, given values of membership functions $\mu_{a}$ and $\mu_{b}$, the amplitudes $a:[0,1] \rightarrow[0,1]$ and $b:[0,1] \rightarrow[0,1]$ are obtained by the following equations $[5,6]$ :

$$
\begin{aligned}
& a=\operatorname{sign}\left(\mu_{a}+\mu_{b}-1\right) \sqrt{\left|\sin ^{2} \frac{\pi}{2} \mu_{a}+\sin ^{2} \frac{\pi}{2} \mu_{b}-1\right|}, \\
& b=\operatorname{sign}\left(\mu_{b}-\mu_{a}\right) \sqrt{\left|\sin ^{2} \frac{\pi}{2} \mu_{b}-\sin ^{2} \frac{\pi}{2} \mu_{a}\right|} .
\end{aligned}
$$

The Hannachi and the $\mathrm{H}^{3}$-model $[5,6]$ include fuzzy representations of the main qubit operators (Pauli operators I, X, Z, Hadamard H operator and negative unitary operator $I$ ) that are used in quantum information theory [8]. The correspondence between quantum and fuzzy operators is the following. Let us denote fuzzy operators by $\widetilde{\mathrm{I}}$, - $\widetilde{\mathrm{I}}, \widetilde{\mathrm{X}}, \widetilde{\mathrm{Z}}$ and $\widetilde{\mathrm{H}}$ in correspondence to the quantum operators. Then an application of the fuzzy operators to a fuzzy state $\widetilde{s}=\left(\mu_{a}, \mu_{b}\right)$ results in the following states $[5,6]$ :

$$
\begin{aligned}
\widetilde{\mathrm{I}}\left(\begin{array}{l}
\mu_{a} \\
\mu_{b}
\end{array}\right) & =\left(\begin{array}{l}
\mu_{a} \\
\mu_{b}
\end{array}\right),-\widetilde{\mathrm{I}}\left(\begin{array}{l}
\mu_{a} \\
\mu_{b}
\end{array}\right)=\left(\begin{array}{c}
1-\mu_{a} \\
1-\mu_{b}
\end{array}\right), \widetilde{\mathrm{X}}\left(\begin{array}{l}
\mu_{a} \\
\mu_{b}
\end{array}\right)=\left(\begin{array}{c}
1-\mu_{a} \\
\mu_{b}
\end{array}\right), \\
\widetilde{\mathrm{Z}}\left(\begin{array}{l}
\mu_{a} \\
\mu_{b}
\end{array}\right) & =\left(\begin{array}{l}
\mu_{b} \\
\mu_{a}
\end{array}\right), \\
\widetilde{\mathrm{H}}\left(\begin{array}{l}
\mu_{a} \\
\mu_{b}
\end{array}\right) & =\left(\begin{array}{l}
\left.\max \left\{0, \min \left\{1, \frac{1}{2}-\mu_{a}+\mu_{b}\right\}\right\}\right\} \\
\left.\min \left\{1, \max \left\{0, \mu_{a}+\mu_{b}-\frac{1}{2}\right\}\right\}\right) .
\end{array}\right.
\end{aligned}
$$

Since the fuzzy state $\widetilde{s}$ is not a vector, its row and column representation are used here interchangeably.

To complete the correspondence between quantum and fuzzy models, Rybalov et al. [11] suggested the reverse fuzzy Hadamard operator $\widetilde{\mathrm{H}}^{R}$ that corresponds to the quantum reverse Hadamard operator. For a fuzzy state $\widetilde{s}=\left(\mu_{a}, \mu_{b}\right)$ this operator is defined as follows:

$$
\widetilde{\mathrm{H}}^{R}\left(\begin{array}{l}
\mu_{a} \\
\mu_{b}
\end{array}\right)=\left(\begin{array}{l}
\min \left\{1, \max \left\{0, \frac{3}{2}-\mu_{a}-\mu_{b}\right\}\right\} \\
\max \left\{0, \min \left\{1, \frac{1}{2}-\mu_{a}+\mu_{b}\right\}\right\}
\end{array}\right) .
$$

The $\mathrm{H}^{3}$-model with the reverse Hadamard operator $\widetilde{\mathrm{H}}^{R}$ is called extended $\mathrm{H}^{3}$-model.

\section{Phase ${ }_{\alpha}$ and not $_{\alpha}$ operators for $\mathrm{H}^{3}$-model}

Both the original and the extended $\mathrm{H}^{3}$-models are rather complex. Below we suggest a simpler fuzzy model that includes two operators, to which the transformations that are provided by the extended $\mathrm{H}^{3}$-model can be reduced.

Let $\widetilde{s}=\left(\mu_{a}, \mu_{b}\right)$ be a fuzzy state and assume that the values of membership functions $\mu_{a}$ and $\mu_{b}$ are obtained according to the $\mathrm{H}^{3}$-model. However, the fuzzy states $\widetilde{s}=\left(\mu_{a}, \mu_{b}\right)$ are not restricted and the suggested operators can be applied for any pair of membership functions $\left(\mu_{a}, \mu_{b}\right)$.

Let $\alpha$ be a real number such that $0 \leq \alpha \leq 1$. For fuzzy state $\widetilde{s}=\left(\mu_{a}, \mu_{b}\right)$ and the number $\alpha$, we define two operators phase ${ }_{\alpha}$ and not $_{\alpha}$ as follows:

$$
\begin{aligned}
& \operatorname{phase}_{\alpha}\left(\begin{array}{l}
\mu_{a} \\
\mu_{b}
\end{array}\right)=\left(\begin{array}{l}
(1-\alpha) \cdot \mu_{a}+\alpha \cdot\left(1-\mu_{b}\right) \\
\alpha \cdot\left(1-\mu_{a}\right)+(1-\alpha) \cdot\left(1-\mu_{b}\right)
\end{array}\right), \\
& \operatorname{not}_{\alpha}\left(\begin{array}{l}
\mu_{a} \\
\mu_{b}
\end{array}\right)=\left(\begin{array}{l}
(1-\alpha) \cdot\left(1-\mu_{a}\right)+\alpha \cdot \mu_{b} \\
\alpha \cdot \mu_{a}+(1-\alpha) \cdot \mu_{b}
\end{array}\right) .
\end{aligned}
$$

It is easy to verify that the operators phase $_{\alpha}$ and not $_{\alpha}$ have the following properties:

$$
\begin{array}{ll}
\text { phase }_{\alpha=0}\left(\begin{array}{l}
\mu_{a} \\
\mu_{b}
\end{array}\right)=\left(\begin{array}{l}
\mu_{a} \\
1-\mu_{b}
\end{array}\right), & \operatorname{not}_{\alpha=0}\left(\begin{array}{l}
\mu_{a} \\
\mu_{b}
\end{array}\right)=\left(\begin{array}{l}
1-\mu_{a} \\
\mu_{b}
\end{array}\right), \\
\text { phase }_{\alpha=1}\left(\begin{array}{l}
\mu_{a} \\
\mu_{b}
\end{array}\right)=\left(\begin{array}{l}
1-\mu_{b} \\
1-\mu_{a}
\end{array}\right), & \operatorname{not}_{\alpha=1}\left(\begin{array}{l}
\mu_{a} \\
\mu_{b}
\end{array}\right)=\left(\begin{array}{l}
\mu_{b} \\
\mu_{a}
\end{array}\right) .
\end{array}
$$

In addition, for the compositions of the operators phase ${ }_{\alpha}$ and not $\alpha$ with any $\alpha \in[0,1]$, one obtains:

$$
\begin{aligned}
& \operatorname{phase}_{\alpha}\left(\operatorname{phase}_{\alpha}\left(\begin{array}{l}
\mu_{a} \\
\mu_{b}
\end{array}\right)\right)=\operatorname{not}_{\alpha}\left(\operatorname{not}_{\alpha}\left(\begin{array}{l}
\mu_{a} \\
\mu_{b}
\end{array}\right)\right)=\left(\begin{array}{l}
\left(2 \alpha^{2}-2 \alpha+1\right) \cdot \mu_{a}+\alpha \cdot(1-\alpha) \\
\left(2 \alpha^{2}-2 \alpha+1\right) \cdot \mu_{b}+\alpha \cdot(1-\alpha)
\end{array}\right), \\
& \text { phase }_{\alpha}\left(\operatorname{not}_{\alpha}\left(\begin{array}{l}
\mu_{a} \\
\mu_{b}
\end{array}\right)\right)=\operatorname{not}_{\alpha}\left(\operatorname{phase}_{\alpha}\left(\begin{array}{l}
\mu_{a} \\
\mu_{b}
\end{array}\right)\right)=\left(\begin{array}{l}
1-\left(2 \alpha^{2}-2 \alpha+1\right) \cdot \mu_{a}-\alpha \cdot(1-\alpha) \\
1-\left(2 \alpha^{2}-2 \alpha+1\right) \cdot \mu_{b}-\alpha \cdot(1-\alpha)
\end{array}\right)= \\
& =\left(\begin{array}{l}
1 \\
1
\end{array}\right)-\text { phase }_{\alpha}\left(\text { phase }_{\alpha}\left(\begin{array}{l}
\mu_{a} \\
\mu_{b}
\end{array}\right)\right)=\left(\begin{array}{l}
1 \\
1
\end{array}\right)-\operatorname{not}_{\alpha}\left(\operatorname{not}_{\alpha}\left(\begin{array}{l}
\mu_{a} \\
\mu_{b}
\end{array}\right)\right) .
\end{aligned}
$$

In particular, for $\alpha=0$ and $\alpha=1$, the compositions of the operators result in the following states:

$$
\begin{aligned}
& \text { phase }_{\alpha=0}\left(\text { phase }_{\alpha=0}\left(\begin{array}{c}
\mu_{a} \\
\mu_{b}
\end{array}\right)\right)=\operatorname{not}_{\alpha=0}\left(\operatorname{not}_{\alpha=0}\left(\begin{array}{c}
\mu_{a} \\
\mu_{b}
\end{array}\right)\right)= \\
& =\text { phase }_{\alpha=1}\left(\text { phase }_{\alpha=1}\left(\begin{array}{c}
\mu_{a} \\
\mu_{b}
\end{array}\right)\right)=\operatorname{not}_{\alpha=1}\left(\text { not }_{\alpha=1}\left(\begin{array}{c}
\mu_{a} \\
\mu_{b}
\end{array}\right)\right)=\left(\begin{array}{c}
\mu_{a} \\
\mu_{b}
\end{array}\right) \text {, } \\
& \text { phase }_{\alpha=0}\left(\text { not }_{\alpha=0}\left(\begin{array}{c}
\mu_{a} \\
\mu_{b}
\end{array}\right)\right)=\operatorname{not}_{\alpha=0}\left(\text { phase }_{\alpha=0}\left(\begin{array}{c}
\mu_{a} \\
\mu_{b}
\end{array}\right)\right)= \\
& =\text { phase }_{\alpha=1}\left(\operatorname{not}_{\alpha=1}\left(\begin{array}{l}
\mu_{a} \\
\mu_{b}
\end{array}\right)\right)=\text { not }_{\alpha=1}\left(\text { phase }_{\alpha=1}\left(\begin{array}{l}
\mu_{a} \\
\mu_{b}
\end{array}\right)\right)=\left(\begin{array}{l}
1-\mu_{a} \\
1-\mu_{b}
\end{array}\right) .
\end{aligned}
$$

Let us apply the defined operators phase ${ }_{\alpha}$ and not $_{\alpha}$ and their compositions with $\alpha=0$ and $\alpha=1$ to the fuzzy states $\widetilde{s}_{1}=(1,1)$, $-\widetilde{s}_{1}=(0,0), \widetilde{s}_{2}=(0,1)$ and $-\widetilde{s}_{2}=(1,0)$, in which the values of membership 
Citation: Rybalov A, Kagan E, Rapoport A, Ben-Gal I (2014) Fuzzy Implementation of Qubits Operators. J Comput Sci Syst Biol 7: 163-168. doi:10.4172/jcsb.1000151

functions are integer (including zero). The implementation of the presented formulas results in the states with integer values of membership functions, as it is shown in Figure 1.

Now, let us present the actions of the operators phase ${ }_{\alpha}$ and not ${ }_{\alpha}$ over the states $\widetilde{s}_{3}=(1 / 2,1),-\widetilde{s}_{3}=(1 / 2,0), \widetilde{s}_{4}=(1,1 / 2)$ and $-\widetilde{s}_{4}=(0,1 / 2)$. For $\alpha=0$ and $\alpha=1$, these operators act as it is shown in Figure 2.

Finally, let us consider the actions of the operators phase ${ }_{\alpha}$ and not $_{\alpha}$ with $\alpha=1 / 2$. Direct calculations show that for the fuzzy states $\widetilde{s}_{1}=(1,1),-\widetilde{s}_{1}=(0,0), \quad \widetilde{s}_{2}=(0,1)$ and $-\widetilde{s}_{2}=(1,0)$ with integer values of membership functions the operators phase p $_{\alpha=1 / 2}$ and $n o t_{\alpha=1 / 2}$ result in the states that lie on the perimemter of the unit square.

However, an application of the operators phase $_{\alpha=1 / 2}$ and not $_{\alpha=1 / 2}$ to the resulting states $\widetilde{s}_{3}=(1 / 2,1),-\widetilde{s}_{3}=(1 / 2,0), \widetilde{s}_{4}=(1,1 / 2)$ and $-\widetilde{s}_{4}=(0,1 / 2)$ with non-integer values of membership functions does not give the original states. The states that correspond to these values are located in the internal part of the unit square instead of its perimeter. To project such states to the perimeter let us define the unique projector $\widetilde{\mathrm{P}}$ such that for any fuzzy state $\left(\mu_{a}^{\prime}, \mu_{b}^{\prime}\right)$ it results in the state $\left(\mu_{a}, \mu_{b}\right)=\widetilde{\mathrm{P}}\left(\mu_{a}^{\prime}, \mu_{b}^{\prime}\right)$, which lies on the closest intersection point of the perimeter, and a line that follows via the point $\left(\mu_{a}^{\prime}, \mu_{b}^{\prime}\right)$

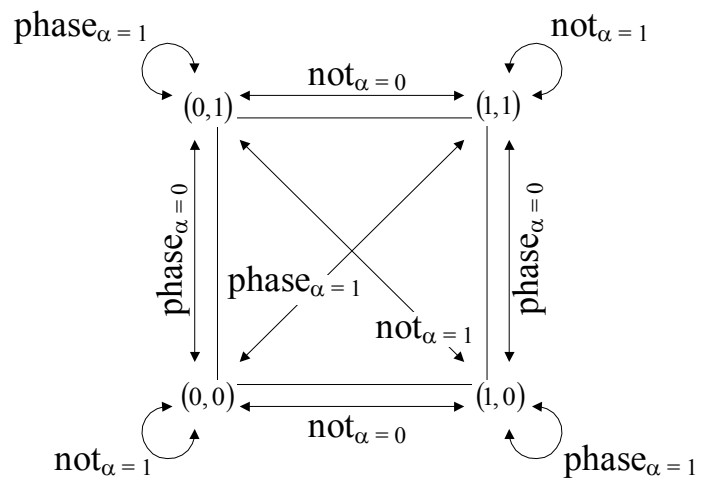

Figure 1: Actions of phase $e_{\alpha}$ and $n o t_{\alpha}$ operators over the fuzzy states with integer values of membership functions.

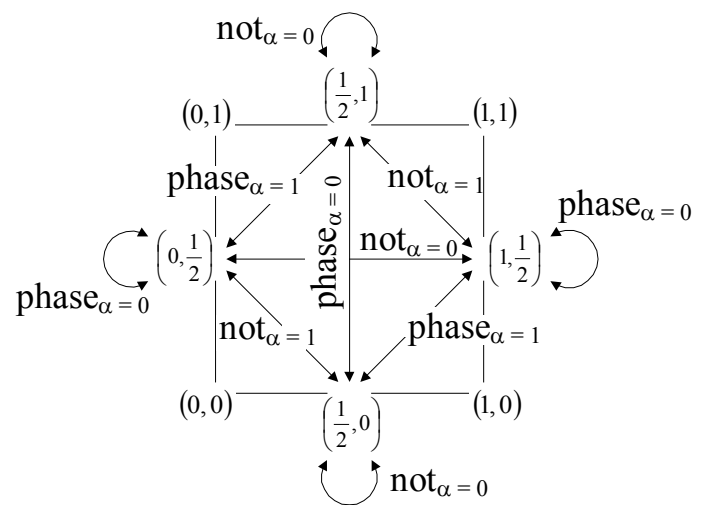

Figure 2: Actions of phase $_{\alpha}$ and not $_{\alpha}$ operators over the fuzzy states with non-integer values of membership functions.

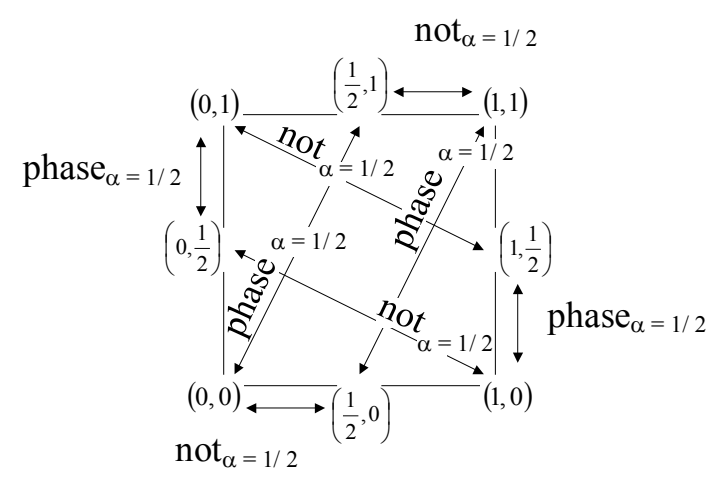

Figure 3: Actions of phase $_{\alpha}$ and not $_{\alpha}$ operators with $\alpha=1 / 2$.

\begin{tabular}{|c|c|c|}
\hline Quantum operator & Extended $\mathbf{H}^{3}$ model & phase $_{\alpha}$ - not $_{\alpha}$ model \\
\hline$I=\left(\begin{array}{ll}1 & 0 \\
0 & 1\end{array}\right)$ & $\mu_{a} \mapsto \mu_{a}$ & phase $_{\alpha}^{2}$ or not $_{\alpha}^{2}$, \\
$\mu_{b} \mapsto \mu_{b}$ & $\alpha=0$ or $\alpha=1$
\end{tabular}

In the table, phase ${ }_{\alpha}^{2}$ and not $_{\alpha}^{2}$ stand for a composition of the operators phase $_{\alpha}^{2}=$ phase $_{\alpha} \circ$ phase $_{\alpha}$ and not $_{\alpha}^{2}=$ not $_{\alpha} \circ$ not $_{\alpha}$, correspondently.

Table 1: Correspondence between quantum, $\mathrm{H}^{3}$ and phase ${ }_{\alpha}-$ not $_{\alpha}$ models.

and a center of the square $(1 / 2,1 / 2)$. The strict meaning of the projector is considered in the next section. The actions of the operators phase ${ }_{\alpha}$ and not $_{\alpha}$ with $\alpha=1 / 2$ (composed with the projector $\widetilde{\mathrm{P}}$, i.e. under the assumption that phase $_{\alpha} \equiv$ phase $_{\alpha} \circ \widetilde{\mathrm{P}}$ and not $_{\alpha} \equiv$ not $\left._{\alpha} \circ \widetilde{\mathrm{P}}\right)$ are shown in Figure 3.

For convenience, the correspondence between quantum and $\mathrm{H}^{3}$ model operators and their representation by the use of phase $e_{\alpha}$ and not $t_{\alpha}$ operators is summarized in Table 1.

The above-presented consideration demonstrates that the suggested phase $e_{\alpha}$ and not ${ }_{\alpha}$ operators completely implement the transformations, which are provided by the extended $\mathrm{H}^{3}$-model that includes fuzzy reverse Hadamard operator $\widetilde{\mathrm{H}}^{R}$.

\section{Uninorm for Phase $e_{\alpha}$ and not ${ }_{\alpha}$ Operators and Projector $\widetilde{\mathrm{P}}$}

Let us clarify the meaning of the above-introduced projecting operator $\widetilde{\mathrm{P}}$. Below we show that this operator provides a monotony 
property of the uninorms that are defined by compositions of the operators phase ${ }_{\alpha}$ and not $_{\alpha}$.

Let us start with the uninorm that is defined over the membership functions. The uninorm $R$ is defined as a mapping $R:[0,1] \times[0,1] \rightarrow[0,1]$ such that for any values of membership functions $\mu_{a}, \mu_{b}, \mu_{c}, \mu_{d} \in[0,1]$, the following properties hold [12]:

commutativity: $R\left(\mu_{a}, \mu_{b}\right)=R\left(\mu_{b}, \mu_{a}\right)$;

monotonicity: $R\left(\mu_{a}, \mu_{b}\right) \geq R\left(\mu_{c}, \mu_{d}\right)$ if $\mu_{a} \geq \mu_{c}$ and $\mu_{b} \geq \mu_{d}$;

associativity: $R\left(\mu_{a}, R\left(\mu_{b}, \mu_{c}\right)\right)=R\left(R\left(\mu_{a}, \mu_{b}\right), \mu_{c}\right)$;

existence of identity: for any $\mu \in[0,1]$ there exists $\varepsilon \in[0,1]$ such that $R(\mu, \varepsilon)=\mu$.

Let us define a similar uninorm $U$ that is applicable to phase $e_{\alpha}$ and $n o t_{\alpha}$ operators, which act on the pairs $\left(\mu_{a}, \mu_{b}\right)$ and result in the pairs $\left(\mu_{a}^{\prime}, \mu_{b}^{\prime}\right), \mu_{a}, \mu_{b}, \mu_{a}^{\prime}, \mu_{b}^{\prime} \in[0,1]$. Below we denote these operators by $A, B, C$ and $D$.

Assume that the uninorm $U(A, B)$ is an operator over the fuzzy states, which is defined as a composition of the operators $A, B$, i.e. $U(A, B)=B \circ A$. Since, as indicated above, it holds true that phase ${ }_{\alpha} \circ$ not $_{\alpha}=$ not $_{\alpha} \circ$ phase $_{\alpha}$, and $\operatorname{phase}_{\alpha}^{2}\left(\mu_{a}, \mu_{b}\right)=\operatorname{not}_{\alpha}^{2}\left(\mu_{a}, \mu_{b}\right)=\left(\mu_{a}, \mu_{b}\right)$, and acting of such uninorm $U$ on the phase $_{\alpha}$ and not $_{\alpha}$ operators meets the commutativity and associativity requirements, and an identity $E=$ phase $_{\alpha} \circ$ phase $_{\alpha}$ (or $E=$ not $_{\alpha} \circ$ not $_{\alpha}$ ) exists.

The consideration of the monotonicity of the uninorm $U$ requires definition of the relations "<" and ">" between the operators. At first, notice that the uninorm $U$ for the operators acts on the pairs of pairs of membership functions and results in the pair of membership functions, i.e.,

$$
U\left(\left(\mu_{a}, \mu_{b}\right),\left(\mu_{c}, \mu_{d}\right)\right) \mapsto\left(\mu_{r}, \mu_{s}\right), \mu_{r}, \mu_{s} \in[0,1] .
$$

In addition, notice, that the pair $(1 / 2,1 / 2)$ represents a fixed point for the operators phase $_{\alpha}$ and not ${ }_{\alpha}$ with any value of parameter $\alpha$. Hence, this point can be considered as a rapper or null-point.

By the use of the point $\tilde{\theta}=(1 / 2,1 / 2)$, the relations "<" and ">" between the operators can be defined as follows. Let $d:\left(\widetilde{s}^{\prime}, \widetilde{s}^{\prime \prime}\right) \rightarrow m \in[0, \infty)$ be a metric defined on the unit square, where $\widetilde{s}^{\prime}=\left(\mu_{a}^{\prime}, \mu_{b}^{\prime}\right), \widetilde{s}^{\prime \prime}=\left(\mu_{a}^{\prime \prime}, \mu_{b}^{\prime \prime}\right)$ and $\mu_{a}^{\prime}, \mu_{b}^{\prime}, \mu_{a}^{\prime \prime}, \mu_{b}^{\prime \prime} \in[0,1]$. Then, for any fuzzy state $\widetilde{s}=\left(\mu_{a}, \mu_{b}\right)$ the distance from this state to the nullpoint $\widetilde{\theta}=(1 / 2,1 / 2)$ is given by the formula:

$$
m\left(\mu_{a}, \mu_{b}\right)=d(\tilde{s}, \widetilde{\theta})=d\left(\left(\mu_{a}, \mu_{b}\right),\left(\frac{1}{2}, \frac{1}{2}\right)\right),
$$

The relations "<" and ">" between the states $\widetilde{s}^{\prime}=\left(\mu_{a}^{\prime}, \mu_{b}^{\prime}\right)$ and $\widetilde{s}^{\prime \prime}=\left(\mu_{a}^{\prime \prime}, \mu_{b}^{\prime \prime}\right)$ are specified by the use of the distances as follows. We say that the state $\widetilde{s}^{\prime}=\left(\mu_{a}^{\prime}, \mu_{b}^{\prime}\right)$ is greater than the state $\widetilde{s}^{\prime \prime}=\left(\mu_{a}^{\prime \prime}, \mu_{b}^{\prime \prime}\right)$ and write $\widetilde{s}^{\prime}>\widetilde{s}^{\prime \prime}$ if in the defined metric $d$ it holds true that

$$
m\left(\mu_{a}^{\prime}, \mu_{b}^{\prime}\right)=d(\widetilde{s}, \widetilde{\theta})>m\left(\mu_{a}^{\prime \prime}, \mu_{b}^{\prime \prime}\right)=d\left(\widetilde{s}^{\prime \prime}, \widetilde{\theta}\right),
$$

and, similarly, we say that $\widetilde{S}^{\prime}$ is less the state $\widetilde{S}^{\prime \prime}$ and write $\widetilde{s}^{\prime}<\widetilde{s}^{\prime \prime}$ if it holds true that

$$
m\left(\mu_{a}^{\prime}, \mu_{b}^{\prime}\right)=d(\widetilde{s}, \widetilde{\theta})<m\left(\mu_{a}^{\prime \prime}, \mu_{b}^{\prime \prime}\right)=d\left(\widetilde{s}^{\prime \prime}, \widetilde{\theta}\right) .
$$

If both $\widetilde{S}^{\prime}>\widetilde{S}^{\prime \prime}$ and $\widetilde{S}^{\prime}<\widetilde{S}^{\prime \prime}$ are satisfied, then we say that the states are equal in the sense of distance from the null-point $\theta$ and write $\widetilde{s}^{\prime}=\widetilde{s}^{\prime \prime}$.

The relations between the operators $A$ and $B$ that act over the fuzzy states, and, correspondently, between the results of the applications of uninorm $U$ are defined by the relations between the resulting states as follows. Let $A\left(\mu_{a}, \mu_{b}\right)=\left(\mu_{a}^{\prime}, \mu_{b}^{\prime}\right)$ and $B\left(\mu_{a}, \mu_{b}\right)=\left(\mu_{a}^{\prime \prime}, \mu_{b}^{\prime \prime}\right)$. Then, we say that $A>B$ if $\left(\mu_{a}^{\prime}, \mu_{b}^{\prime}\right)>\left(\mu_{a}^{\prime \prime}, \mu_{b}^{\prime \prime}\right)$ and $A<B$ if $\left(\mu_{a}^{\prime}, \mu_{b}^{\prime}\right)<\left(\mu_{a}^{\prime \prime}, \mu_{b}^{\prime \prime}\right)$.

Finally, let $A, B, C$ and $D$ be fuzzy operators, and let $U(A, B)=B \circ A=\left(\mu_{a}, \mu_{b}\right)$ and $U(C, D)=D \circ C=\left(\mu_{c}, \mu_{d}\right)$. Then, we say that

$$
\begin{aligned}
& U(A, B)>U(C, D) \text { if }\left(\mu_{a}, \mu_{b}\right)>\left(\mu_{c}, \mu_{d}\right), \text { and } \\
& U(A, B)<U(C, D) \text { if }\left(\mu_{a}, \mu_{b}\right)<\left(\mu_{c}, \mu_{d}\right) .
\end{aligned}
$$

Now, let us consider the role of the projecting operator $\widetilde{\mathrm{P}}$. Assume that the metric $d$ is a Manhattan metric, i.e.

$$
m\left(\mu_{a}, \mu_{b}\right)=d(\tilde{s}, \tilde{\theta})=\left|\mu_{a}-\frac{1}{2}\right|+\left|\mu_{b}-\frac{1}{2}\right| .
$$

Let $\left(\mu_{a}, \mu_{b}\right)$ be a state, and assume that fuzzy operators $A, B, C$ and $D$ without projector $\widetilde{\mathrm{P}}$ act as follows:

$$
\begin{aligned}
& A\left(\mu_{a}, \mu_{b}\right)=\left(\frac{1}{8}, \frac{7}{8}\right), \quad B\left(\mu_{a}, \mu_{b}\right)=\left(\frac{7}{8}, \frac{7}{8}\right), \quad C\left(\mu_{a}, \mu_{b}\right)=\left(0, \frac{1}{2}\right), \\
& D\left(\mu_{a}, \mu_{b}\right)=\left(1, \frac{1}{2}\right) .
\end{aligned}
$$

Then, by the use of the projector $\widetilde{\mathrm{P}}$, i.e., under an assumption that $A \equiv A \circ \widetilde{\mathrm{P}}, B \equiv B \circ \widetilde{\mathrm{P}}, C \equiv C \circ \widetilde{\mathrm{P}}$ and $D \equiv D \circ \widetilde{\mathrm{P}}$, one obtains:

$$
A\left(\mu_{a}, \mu_{b}\right)=(0,1), \quad B\left(\mu_{a}, \mu_{b}\right)=(1,1),
$$

while the results of the operators $C$ and $D$ are still the same. Hence the inequalities $A>C$ and $B>D$ hold both for original results and for the states, which are obtained by the use of the projector $\widetilde{\mathrm{P}}$.

Direct calculations show that for the operators $A, B, C$ and $D$ without projector $\widetilde{\mathrm{P}}$ it follows that

$$
m(U(A, B))=m\left(A\left(\frac{7}{8}, \frac{7}{8}\right)\right) \leq \frac{3}{4} \text { and } m(U(C, D))=m\left(C\left(1, \frac{1}{2}\right)\right) \leq 1 .
$$

Thus, for the operators without projector $\widetilde{\mathrm{P}}$ in spite of the inequalities $A>C$ and $B>D$, the relation $U(A, B)>U(C, D)$ that is required by monotonicity, in general, is not satisfied.

However, similar considerations that include the projector $\widetilde{\mathrm{P}}$ result in the following inequalities $m(U(A, B)) \leq 1$ and $m(U(C, D)) \leq 1$. Notice that both $A\left(\mu_{a}, \mu_{b}\right)=(0,1)$ and $B\left(\mu_{a}, \mu_{b}\right)=(1,1)$ lie on the perimeter of the unit square, i.e. they are boundary states. Moreover, the results of the compositions $U(A, B)=B \circ A$ and $U(C, D)=D \circ C$ are also boundary states. Then, according to definitions of the operators phase $_{\alpha}$ and not $_{\alpha}$, the transformations of the states over the boundary of the unit square are continuous. Hence, from the inequalities $A>C$ and $B>D$ it follows that $U(A, B)>U(C, D)$, as it is required by the 
monotonicity property of the uninorm. This property of the suggested operators allows their consideration in the framework of general fuzzy operators.

\section{Implementation of the Suggested Model and Numerical Analysis}

The suggested model that is based on phase ${ }_{\alpha}$ and not ${ }_{\alpha}$ operators, as well as extended $\mathrm{H}^{3}$-model, were implemented in a mobile robot controller, and for both models comparative numerical simulations and field experiments were carried out. As indicated above, the detailed consideration of the experiments and simulation results is presented in our work [11].

The correspondence between the robot's states and its orientation has been specified according to the quantum "spin orientations" as follows:

north, $0^{0} \equiv 360^{\circ}: \widetilde{s}_{1}=(1,1),-\widetilde{s}_{1}=(0,0)$, south, $180^{\circ}: \widetilde{s}_{2}=(0,1)$, $-\widetilde{s}_{2}=(1,0)$,

east, $270^{\circ}: \widetilde{s}_{3}=\left(\frac{1}{2}, 1\right),-\widetilde{s}_{3}=\left(\frac{1}{2}, 0\right)$, west, $90^{\circ}: \widetilde{s}_{4}=\left(1, \frac{1}{2}\right)$, $-\widetilde{s}_{4}=\left(0, \frac{1}{2}\right)$

The implemented sequence of actions, in the terms of extended $\mathrm{H}^{3}$ model, has the following form:

Initial state $\rightarrow \widetilde{\mathrm{H}} \rightarrow \widetilde{\mathrm{H}} \rightarrow \widetilde{\mathrm{H}}^{R} \rightarrow \widetilde{\mathrm{H}}^{R} \rightarrow \widetilde{\mathrm{H}}^{R} \rightarrow \widetilde{\mathrm{H}} \rightarrow \widetilde{\mathrm{H}} \rightarrow \widetilde{\mathrm{H}} \rightarrow \widetilde{\mathrm{H}}^{R} \rightarrow \widetilde{\mathrm{H}}^{R} \rightarrow \widetilde{\mathrm{H}}^{R} \rightarrow \widetilde{\mathrm{H}}$

According to this sequence, the robot conducts the following actions:

- moving one step forward;

- turning to the right (direct Hadamard operator);

- moving one step forward;

- turning to the left (application of the direct Hadamard operator over eastern orientation of the robot turns the robot to the left returning it to the initial orientation;

- moving one step forward;

- turning to the right (in contrast to the direct Hadamard operator, reverse Hadamard operator turns the robot left from its initial orientation);

- moving one step forward,

and so on up to the last step when the robot returns to its initial state. The obtained trajectory is simple enough for analysis, and, at the same time, represents all possible turns of the robot, so that all transitions between the states can be tested.

In the field experiments, the mobile robot was programmed so that it implemented the presented sequence of actions according to either the extended $\mathrm{H}^{3}$-model or the suggested model based on phase ${ }_{\alpha}$ and not ${ }_{\alpha}$ operators. An example of the obtained trajectories of the robot, while at the initial state it was oriented to the north, are shown in Figure 4 .

The Figure demonstrates the difference between turns that are governed by the extended $\mathrm{H}^{3}$-model and the suggested model based on phase ${ }_{\alpha}$ and not $_{\alpha}$ operators. Notice that the turns of the robot controlled by phase $e_{\alpha}$ and not $t_{\alpha}$ model differ from the turns that were

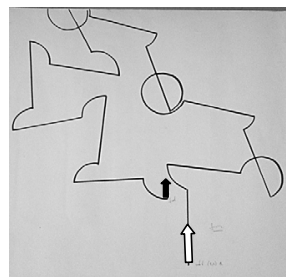

Extended $\mathrm{H}^{3}$-model

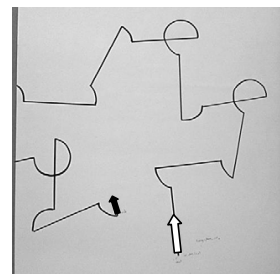

phase $_{\alpha}$ and not $t_{\alpha}$ model
Figure 4: Trajectories of the robot movements controlled by the extended $\mathrm{H}^{3}$-model and by the model based on of phase ${ }_{\alpha}$ and not $_{\alpha}$ operators. White arrow denotes a starting position and orientation of the robot, and a black arrow denotes its final position and orientation.
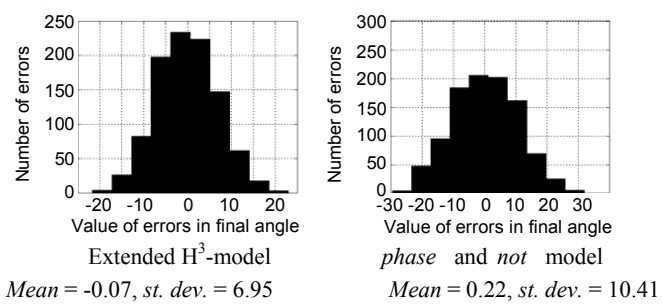

(a): $1 \%$ turns' errors
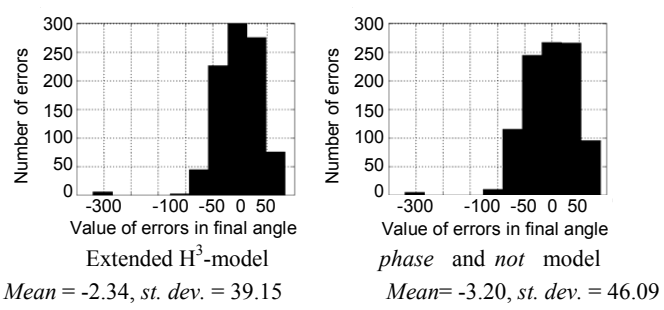

(b): $5 \%$ turns' errors
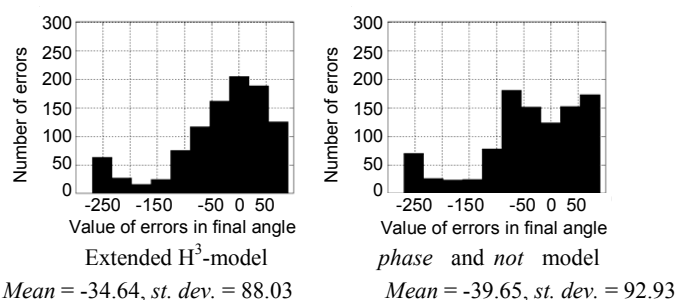

(c): $10 \%$ turns' errors.

Figure 5: Histograms of the final angle errors with different percentage of turn's errors.

previously obtained for the model of quantum control [11], so the topology of the states space for the phase ${ }_{\alpha}$ and not ${ }_{\alpha}$ model differs from both the extended $\mathrm{H}^{3}$ and the quantum models.

Numerical analysis was focused on the errors of the final angle that reflect the turns' errors. The above-indicated sequence of direct and reverse Hadamard operators was implemented numerically, while after each application of the operator a random error to the resulting state was added. There were implemented three values of errors: $1 \%$ error, $5 \%$ error and $10 \%$ error. The $1 \%$ error corresponds to the adding to the robot's orientation a randomly chosen angle from the interval $\left(-3.6^{0}, 3.6^{\circ}\right)$, the $5 \%$ error means adding a randomly chosen 
Citation: Rybalov A, Kagan E, Rapoport A, Ben-Gal I (2014) Fuzzy Implementation of Qubits Operators. J Comput Sci Syst Biol 7: $163-168$. doi:10.4172/jcsb.1000151

angle form the interval $\left(-18^{0}, 18^{\circ}\right)$, and $10 \%$ error corresponds to the addition of the angle randomly chosen from the interval $\left(-36^{\circ}, 36^{\circ}\right)$. For each interval, the simulation was trialed 1000 times and the differences between the obtained and expected final orientations, i.e., the errors in final orientation angles were logged.

Histograms of the orientation errors in the final angle of the robots are shown in Figure 5. The histograms in Figure 5a correspond to the $1 \%$ errors in the robot's turns; the histograms in Figure 5b-to 5\% turns' errors; and the histograms in Figure 5c-to a 10\% turns' errors.

By the use of the Jarque-Bera test with significance level 0.01 , it was found that in the case of $1 \%$ errors, the distributions of errors for both models of control are approximately normal, while in cases of $5 \%$ and $10 \%$ errors the distributions of errors can not be considered as normal. A series of $t$-tests with a significance level of 0.01 showed that the difference between the resulting errors is insignificant. Following the Kolmogorov-Smirnov test with a significance level 0.01, in all three cases of errors the obtained samples correspond to different continuous distributions.

The obtained results confirm our expectation that the model that is based on phase ${ }_{\alpha}$ and not ${ }_{\alpha}$ operators acts differently in comparison to the extended $\mathrm{H}^{3}$-model, while the results of application of the suggested operators are equivalent. As indicated above, the difference between the actions of the extended $\mathrm{H}^{3}$-model and the model based on phase $_{\alpha}$ and not ${ }_{\alpha}$ operators is provided by different topologies of the states space.

\section{Conclusion}

In the paper, we suggested a system of fuzzy operators that implements the operations over qubits. The system includes two parameterized phase $e_{\alpha}$ and not $_{\alpha}$ operators that act on the states obtained by the extended $\mathrm{H}^{3}$-model. The transformations of the states that correspond to the operators of the extended $\mathrm{H}^{3}$-model are determined by compositions of the defined phase ${ }_{\alpha}$ and not $_{\alpha}$ operators. For the suggested operators, the operational uninorm was defined and analyzed, what introduces the suggested operators into general fuzzy logic approach to quantum computations.

The suggested system was illustrated by implementation for a mobile robot control and was analyzed by numerical simulations. Comparison of the control based on the suggested control demonstrated that the results of the actions of the suggested minimal fuzzy system (that includes two operators) are equivalent to the corresponding results obtained by the extended $\mathrm{H}^{3}$-model (that includes six operators).

The obtained results can support a development of fuzzy logic tools for the models of quantum information theory.

\section{Acknowledgement}

We are thankful to Moshe Israel and Yaniv Reginiano for fruitful discussions. The research was conducted in the CIM and Process Control Lab at Tel Aviv University.

\section{References}

1. Birkhoff G, Von Neumann J (1936) The logic of quantum mechanics. Annals Math 37: 823-843.

2. G Cattaneo, Chiara MLD, Giuntini R, Paoli F (2009) Quantum logicandnonclassicallogics. Quantum Logic and Quantum Structures 127-226.

3. Nielsen MA, Chuang IL (2000) Quantum Computation and Quantum Information. Information and the Natural Sciences 1-29.

4. Aerts D, Durt T, Van Bogaert B (1993) A physical example of quantum fuzzy sets and the classical limit. Tatra Mt Math Publ 1: 5-15.

5. Hannachi MS, Hatakeyama Y, Hirota K (2007) Emulating quantum interference and quantum associative memory using fuzzy qubits. Proc IEEE IntConf Computational Cybernetics 39-45.

6. Hannachi MS, Hatakeyama Y, Hirota K (2007) Emulating qubits with fuzzy logic J Advanced Computational Intelligence and Intelligent Informatics 11: 242-249.

7. Kagan E, Ben-Gal I (2011) Navigation of quantum-controlled mobile robots. Recent Advances in Mobile Robotics 311-326.

8. Pykacz J (1992) Fuzzy set ideas in quantum logics. Int J TheorPhys 31: 1767 1783.

9. Kovačić Z, Bogdan S (2006) Fuzzy Controller Design Theory and Applications Automation and Control Engineering 1-416.

10. Rigatos GG, Tzafestas SG (2002) Parallelization of a fuzzy control algorithm using quantum computation. IEEE Trans Fuzzy Systems 10: 451-460.

11. Rybalov, Kagan E, Manor Y, Ben-Gal I (2010) Fuzzy model of control fo quantum-controlled mobile robots. IEEE $26^{\text {th }}$ Conv Electrical and Electronics Engineers in Israel.

12. Yager RR, Rybalov A (1996) Uninorm aggregation operators. Fuzzy Sets and Systems 80: 111-120. 